\title{
Non-Maxwellian electron distributions in time-dependent simulations of low-Z materials illuminated by a high-intensity X-ray laser
}

\author{
Alberto G. de la Varga ，Pedro Velarde , François de Gaufridy • , David Portillo , \\ Manuel Cotelo , Alfonso Barbas , Agustín González , Philippe Zeitoun
}

\begin{abstract}
A B S T R A C T
The interaction of high intensity X-ray lasers with matter is modeled. A collisional-radiative timedependent module is implemented to study radiation transport in matter from ultrashort and ultraintense X-ray bursts. Inverse bremsstrahlung absorption by free electrons, electron conduction or hydrodynamic effects are not considered. The collisional-radiative system is coupled with the electron distribution evolution treated with a Fokker-Planck approach with additional inelastic terms. The model includes spontaneous emission, resonant photoabsorption, collisional excitation and de-excitation, radiative recombination, photoionization, collisional ionization, three-body recombination, autoionization and dielectronic capture. It is found that for high densities, but still below solid, collisions play an important role and thermalization times are not short enough to ensure a thermal electron distribution. At these densities Maxwellian and non-Maxwellian electron distribution models yield substantial differences in collisional rates, modifying the atomic population dynamics.
\end{abstract}

\section{Introduction}

Currently collisional-radiative atomic models are the preferred method to treat ionization balance plasmas that are not in equilibrium. When implementing a collisional-radiative model is necessary to employ approximations, either in the treatment or grouping of atomic levels to be considered or in the models employed in the computation of the transition rates connecting these levels [1-3]. In order to simplify computations, most timedependent simulation codes assume a thermal distribution for the free electrons when obtaining collisional rates. However, under certain conditions the electron distribution is not Maxwellian. Some groups have successfully treated this problem in the past [47] with different approaches, here we are interested in modeling $\mathrm{x}$-ray laser-matter interaction, in particular here we treat free electron laser (XFEL) $[8,9]$ and plasma-based high intensity $\mathrm{X}$-ray lasers $[10,11]$ interacting with matter. In this regime materials are illuminated by a monochromatic X-ray source with photon energies up to several keV that are able to photoionize inner shells electrons. These photoionized electrons may have high energies, which depend on the difference between photon energy and the ionization potential of the ionized shell, and if inner shells do ionize, will produce Auger electrons with energies on the order of hundreds of eV. Importantly the collision frequency of high energy electrons is lower than low energy electrons, meaning that high energy electrons, so that high energy electrons will have longer thermalization times. Thus, ignoring the evolution of the electron distribution may lead to substantial differences in collisional rates and hence predict an incorrect evolution of the ionic populations when the dynamics are driven by collisional processes.

We expect inverse bremsstrahlung absorption by free electrons, electron conduction or hydrodynamic effects to be negligible. At first hand these assumptions seem reasonable. Due to the nonequilibrium nature of the problem free electrons are created with high energies which, in addition to the high energy photons from the laser pulse, produces little resonant free-electron absorption that comes mainly from low energy electrons. The short time scale employed leads in principle to small hydrodynamic and electron conduction effects [9]. The influence of this effects will be studied in future versions to check our assumptions. 
Further, there is no treatment of escaped or trapped electrons [12], all electrons are considered trapped and contribute to the average free electron energy and collision rates. Energy exchange involving ions, free electrons and the x-ray pulse is computed in each timestep to check the consistency of the model and to obtain the equivalent thermalized free electron temperature.

In Sec. 2 we present the atomic and electron kinetics models implemented, simulation results of the Ne case [8] are discussed in Sec. 3 and conclusions are presented in Sec. 4.

\section{Theory}

Recently the LTE opacity code bigbart [13] was extended to treat Non-LTE plasmas through a collisional-radiative model. This model includes the processes of spontaneous emission and resonant photoabsorption, collisional excitation and de-excitation, radiative recombination and photoionization, collisional ionization and three-body recombination, autoionization and dielectronic capture. The linear system to be solved is

$$
\frac{\mathrm{d} n_{i}}{\mathrm{~d} t}=-n_{i} \sum_{j \neq i}^{M} R_{i j}+\sum_{j \neq i}^{M} n_{j} R_{j i} \quad i=1, \cdots, M
$$

where $n_{i}$ is the population density of atomic state $i n=\left(n_{1}, \cdots, n_{M}\right), R_{i j}$ and $R_{j i}$ are rates connecting different atomic states and $M$ is the total number of atomic states considered. The steady-state solver, i.e., $(\mathrm{dn} /$ $d t=0$ ), uses as input an electronic temperature $t_{e}$ and density $n_{e}$ to compute rates between atomic states. Rates of certain processes and their inverses are obtained through the detailed balance principle with the relativistic atomic package FAC [14]. Relativistic configurations are grouped in non-relativistic atomic states assuming equilibrium within the non-relativistic configuration at temperature $t_{e}$.

Some modifications and extensions have been made to the code to adapt it to time-dependent atomic computations. First, grouping of atomic states has been extended to the superconfiguration approach [15], where states are identified by the number of electrons in each shell (e.g. $\mathrm{K}^{2} \mathrm{~L}^{8} \mathrm{M}^{2}$ equals 2 electrons in $\mathrm{K}$ shell, 8 in the $\mathrm{L}$ shell and 2 in the $\mathrm{M}$ shell) known as a Layzer complex. This allows faster calculation of atomic populations by reducing the number of states treated.

Second, in addition to FAC atomic data some alternative models have been implemented to allow faster computation of rates and atomic levels. Screened hydrogenic levels with principal quantum number dependence $[16,17]$ were included. All processes between complexes can be computed under the hydrogenic approximation, spontaneous emission [17], collisional excitation [18], photoionization [19], collisional ionization [20] and autoionization [21]. Collisional ionization may be calculated with a second alternative model from Ref. [22]. This model consists of Coulomb-Born exchange (CBE) fits that allow fast computation of cross-sections and Maxwellian averaged rates between nl-averaged (non-relativistic) and n-averaged (complex) configurations. We included a fit to differential cross-sections depending on the energy of the ejected electron, useful in obtaining integrated three-body recombination rates with non-maxwellian electron distributions [5]. We further included a second alternative model for collisional excitation from Ref. [23] based on the plane-wave born approximation (PWBA) named JJATOM. The reason to do this is that the cross-sections computed with JJATOM compare well with distorted-wave approximation (DWA) cross-sections generated by FAC and allows faster computation of the cross-sections. We obtain superconfiguration rates from FAC by averaging rates between levels belonging to the initial $(\Xi)$ and final $\left(\Xi^{\prime}\right)$ superconfiguration, which is done because superconfigurations with more than two open shells may be composed of thousands to hundred of thousands relativistic configurations.
$R_{\Xi \rightarrow \Xi^{\prime}}=\frac{\sum_{a \in \Xi} \sum_{b \in \Xi^{\prime}} g_{a} \exp \left(-\frac{E_{a}}{k T_{e}}\right) R_{a b}}{\sum_{a \in \Xi} g_{a} \exp \left(-\frac{E_{a}}{k T_{e}}\right)}$

where $g_{a}$ and $E_{\alpha}$ are the statistical weight and energy of level $a$, and $R_{a b}$ is the rate connecting levels $a$ and $b$. Although the DWA algorithm in FAC is quite fast, the number of collisional excitations in multiplyexcited superconfigurations is intractable. We have found it best to resort to models like JJATOM and compute cross-sections with FAC wave functions from average relativistic configurations (e.g. $\mathrm{K}^{2} \mathrm{~L}^{8} \mathrm{M}^{2}$ equals $\left.1 \mathrm{~s}_{+}^{2} \cdot 2 \mathrm{~s}_{+}^{2} \cdot 2 \mathrm{p}_{-}^{2} \cdot 2 \mathrm{p}_{+}^{4} \cdot 3 \mathrm{~s}_{+}^{0.2} \cdot 3 \mathrm{p}_{-}^{0.2} \cdot 3 \mathrm{p}_{+}^{0.4} \cdot 3 \mathrm{~d}_{-}^{0.4} \cdot 3 \mathrm{~d}_{+}^{0.6}\right)$. Stimulated spontaneous emission and radiative recombination rates are calculated with formulas from Ref. [24].

The collisional-radiative model is coupled to the electron distribution function through collisional rates, computed from cross-sections and the electron distribution

$R_{i j}=\int_{0}^{\infty} \sqrt{\frac{2 E}{m_{e}}} \sigma_{i j}(E) f(E) \mathrm{d} E$

where $\sqrt{2 E / m_{e}}$ is the electron velocity with energy $E, m_{e}$ is the electron mass, $\sigma_{i j}$ is the cross-section for the process between states $i$ and $j$, and $f$ is the electron energy distribution. The electron distribution is in units of $\left(\mathrm{eV}^{-1} \mathrm{~cm}^{-3}\right)$ and is discretized into $L$ logarithmic energy bins $\boldsymbol{f}=\left(f_{1}, \cdots f_{L}\right)$. Its temporal evolution is treated in the Fokker-Planck (FP) approximation [6] with additional inelastic terms.

$\frac{\partial \boldsymbol{f}}{\partial t}=-\frac{\partial}{\partial E}(a \boldsymbol{f})+\frac{1}{2} \frac{\partial^{2}}{\partial E^{2}}(D \boldsymbol{f})+S+I(\boldsymbol{f})$

where $a$ and $D$ are the energy exchange and diffusion coefficients, $S$ is the source of electrons (photo and auger electrons) and $I(f)$ is an inelastic term that includes all creation and destruction of electron population in each energy bin due to collisions with ions.

The energy exchange and diffusion coefficients depend on the electron energy and are integrated over the temporal electron distribution [6]

$a(E)=\int_{0}^{\infty} a\left(E, E_{1}\right) f\left(E_{1}\right) \mathrm{d} E_{1}$

$D(E)=\int_{0}^{\infty} D\left(E, E_{1}\right) f\left(E_{1}\right) \mathrm{d} E_{1}$

The time-dependent atomic populations $(d \boldsymbol{n} / d t \neq 0)$ are obtained solving the ODE initial value problem with the CVODE solver from Sundials [25]

$\frac{\partial \boldsymbol{n}}{\partial t}=F(t, \boldsymbol{n}, \boldsymbol{f}), \quad \boldsymbol{n}\left(t_{0}\right)=\boldsymbol{n}_{0}$

Finally, for each timestep we compute the average electron energy from the average electron energy density $\rho_{E}$ and the electron density $n_{e}$. From the average energy we can calculate the equivalent thermalized electron temperature

$t_{e}^{t h}=\frac{2}{3}\left\langle E_{a v}\right\rangle=\frac{2}{3} \frac{\rho_{E}}{n_{e}}=\frac{2}{3} \frac{\int_{0}^{\infty} E f(E) \mathrm{d} E}{n_{e}}$

In the simulations performed FAC atomic data was used for all transitions considered except for the collisional ionization where fitted $\mathrm{CBE}$ cross-sections from [22] were used. For collisional 
excitation we used FAC wave functions to compute cross-sections with the JJATOM model [23] as explained above.

\section{Results}

We have chosen to simulate case 3 from Ref. [8]. In this experiment $\mathrm{Ne}$ atoms, at a density of $10^{19} \mathrm{at} / \mathrm{cm}^{3}$, were irradiated with high intensity X-ray bursts. Neon was chosen because it exhibits notable changes in its electronic response in the photon energy range achievable during the experiments, i.e., from 800 to $2000 \mathrm{eV}$. Binding energy of $1 \mathrm{~s}$ electrons vary from $870 \mathrm{eV}$ in neutral Ne to $1360 \mathrm{eV}$ in H-like Ne. Depending on the energy of the X-ray beam the different ionization mechanisms (valence L-shell ionization, inner K-shell ionization and Auger decay following an inner shell vacancy creation) produce different ion population evolutions. In our case we perform simulations at $2 \mathrm{keV}$ photon energy, well above the $\mathrm{K}$ edge of $1.36 \mathrm{keV}$ in $\mathrm{H}$-like $\mathrm{Ne}$. This way we can exclude resonant photoabsorption to simplify the computations. We also excluded three-body recombination, again to simplify the calculation of the inelastic terms in the FP model.

In addition to the experiment's density taken as $10^{19}$ we performed simulations at a higher density of $10^{22}$, below solid density. In this way we can compare results obtained from a more exact treatment of electron distribution and the thermalization approximation (Maxwellian abbreviated MXW) at different densities with different thermalization times.

\subsection{Experiment density: $10^{19}$}

In Fig. 1 we plot the electron distribution evolution at the experiment density of $10^{19} \mathrm{at} / \mathrm{cm}^{3}$. Both FP and MXW distributions are plotted with the FP evolution of the electron density versus the time on the base.

In the FP model the electron distribution remains nonMaxwellian throughout the pulse length, with two visible 'bumps' or high energy components due to Auger and photoelectrons. Auger and photoelectrons are born with energies from hundreds of eV to almost two keV. Table A.1 shows KLL auger decay rates and energies of the ejected electron in the process. Auger electrons have energies ranging from 674 to $885 \mathrm{eV}$, and are produced at similar rates as $\mathrm{K}$ and $\mathrm{L}$ shell ionized electrons together. This can be seen in Fig. 2 where the ratio $\eta$ of total rates for autoionization $\eta^{\mathrm{AI}}$ and collisional ionization $\eta^{\mathrm{CI}}$, divided by the photoionization total rate, are plotted. The total autoionization rate is of the order of magnitude of photoionization throughout the pulse

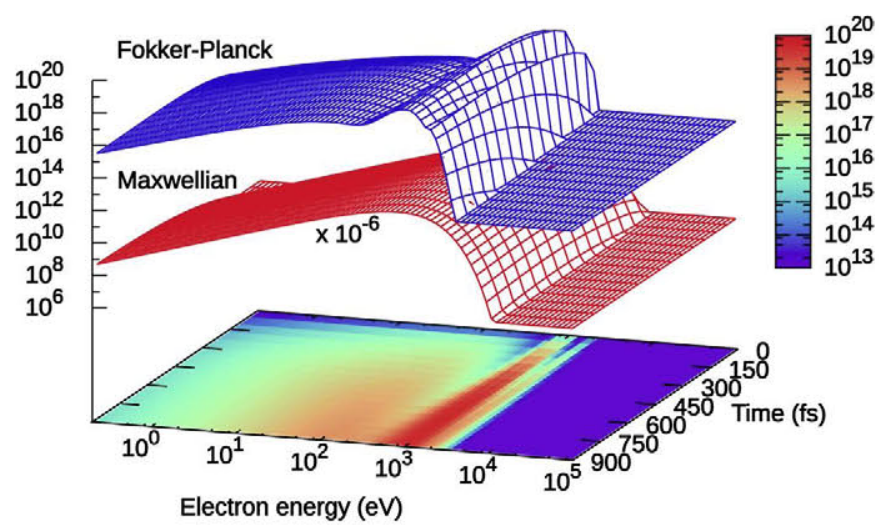

Fig. 1. Electron distribution $\left(\mathrm{eV}^{-1} \mathrm{~cm}^{-3}\right)$ at the experimental density of $10^{19} \mathrm{at} / \mathrm{cm}^{3}$ calculated with the FP and MXW models. The two-dimensional map at base corresponds to the FP model.

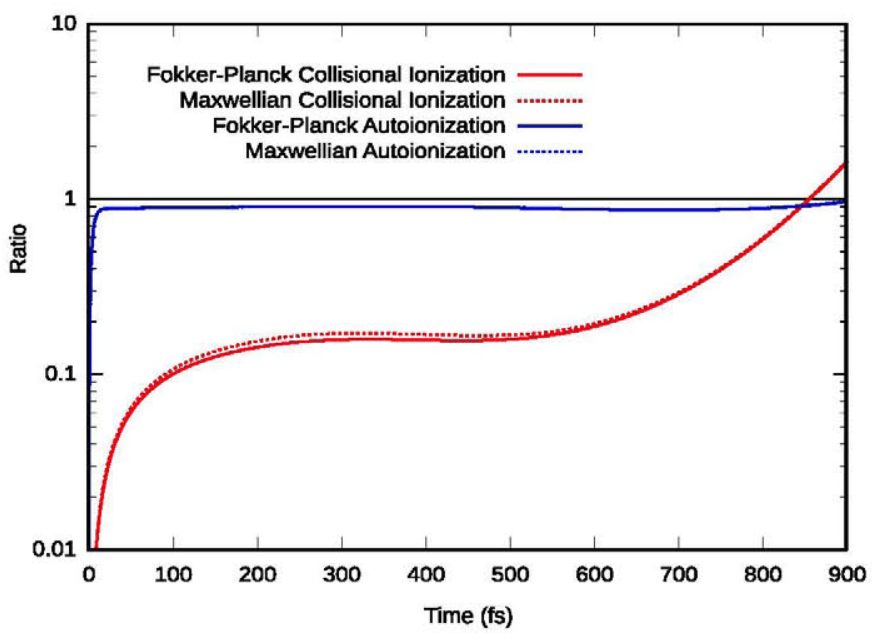

Fig. 2. Ratio of collisional ionization and autoionization total rate to the photoionization total rate at $10^{19} \mathrm{at} / \mathrm{cm}^{3}$ for the FP and MXW models.

duration, competing in the production of high-energy free electrons, repopulating the $\mathrm{K}$ shell and allowing the ejection of new $\mathrm{K}$ electrons.

Photoionization cross-sections and ejected electrons energies for the $K$ and $L$ shell are shown in Tables A.2 and A.3 respectively. Absorption of a photon of $2 \mathrm{keV}$ by a $\mathrm{K}$ electron is at least an order of magnitude higher than the absorption from the $\mathrm{L}$ shell. Autoionization rates depend on the production of $\mathrm{K}$ holes through the absorption of a photon in the $\mathrm{K}$ shell, the dominance of $\mathrm{K}$ absorption over $\mathrm{L}$ shell absorption keeps the ratio between autoionization and photoionization constant with similar rates for depopulation/repopulation mechanism of the $\mathrm{K}$ shell.

At these electron densities collisional ionization hardly affects the dynamics of the atomic states. It is only important at the end of the pulse when the incident intensity decays, due to the temporal Gaussian shape, and a stabilized electron density of $4.47 \times 10^{19}$ and an average ionization of $\bar{Z}=4.47$. Although the electron distribution is clearly non-Maxwellian, the low collisional contribution, as seen in Fig. 2, prevents collisional ionization from being a competitor in the depopulation of K- and L-shells. The MXW model increases the overlap of the electron distribution with the collisional ionization cross-sections of $\mathrm{L}$ shell electrons, slightly increasing its ratio $\eta^{\mathrm{Cl}}$ compared to the FP model. Therefore, the collisional processes can be neglected when treating the atomic dynamics when modeling of experiments [8] for high intensity and at densities low enough to keep collisional processes negligible. Considering thermalization does not significantly modify the evolution of the sample temperature and density, see Fig. 3, the evolution of the sample is dominated by photoionization and autoionization and collisional processes have a low impact. Electrons are born with energies ranging from 650 to $1950 \mathrm{eV}$, approximately $10-15 \%$ of these electrons become low energy electrons, see Fig. 2, through collisional ionization which thermalize quite fast. The steady reduction in the electron equivalent temperature is due to the increase of the ionization threshold with the degree of ionization of the ionizing ions.

\subsection{Higher density case}

In order to evaluate the influence of the simplifications usually assumed in laser-matter interaction simulation codes, we performed a simulation with the parameters of the $2 \mathrm{keV}$ case in the Ne 


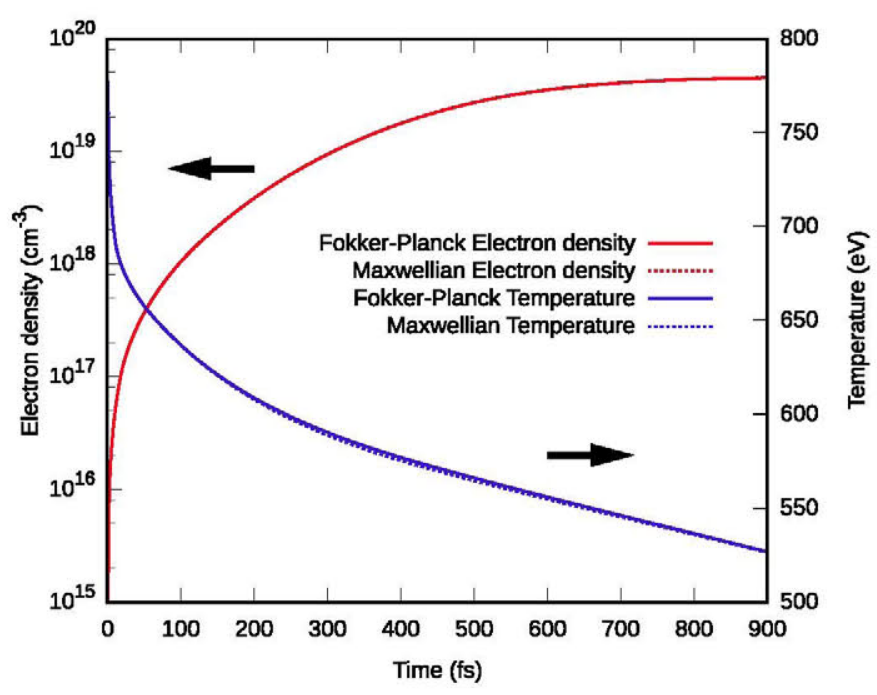

Fig. 3. Electron density and temperature evolution at $10^{19} \mathrm{at} / \mathrm{cm}^{3}$ for the FP and MXW models.

experiment but at higher density. Fig. 4 shows the evolution of the sample as computed with the FP and MXW models for the electron distribution. It can be seen that the high-energy component in the FP model is a fraction of the total electron density when compared to the experimental density case, but is not negligible. Thermalization of high-energy electrons takes at least $600 \mathrm{fs}$ and full thermalization only happens by the end of the pulse when the incident intensity has decreased.

At a density three orders of magnitude higher than the experiment the high energy electrons reduce their energy through collisional ionization at a faster rate, producing two lower energy electrons. This reduces the energy density $\rho_{E}$ of free electrons with an increase in the free electron density $n_{e}$. In the FP model, the initial equivalent thermalized temperature (Eq. (8)) of $778 \mathrm{eV}$ is reduced to a minimum of $11.2 \mathrm{eV}$ at $100 \mathrm{fs}$ by this mechanism. At this point $\eta^{\mathrm{CI}}$ reaches its maximum, see Fig. 5, photoionization and autoionization rates increase with the pulse intensity, inducing a steady rise of the equivalent temperature, as shown in Fig. 6.

The higher electron density accelerates the sample's ionization thanks to the collisional ionization contribution. In the simulation with the experimental conditions it takes $300 \mathrm{fs}$ to reach an average ionization of $\bar{Z}=1$, see Fig. 3; however, in the high density case

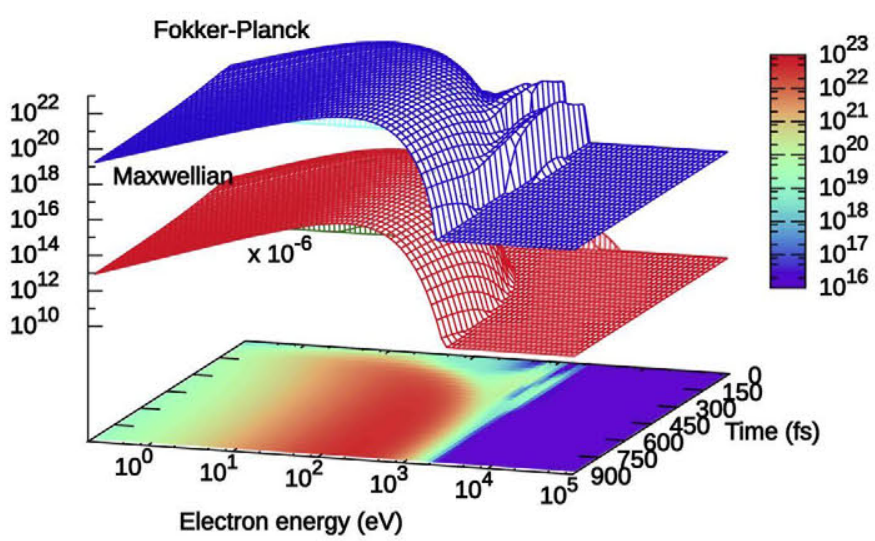

Fig. 4. Electron distribution $\left(\mathrm{eV}^{-1} \mathrm{~cm}^{-3}\right)$ at the higher density of $10^{22} \mathrm{at} / \mathrm{cm}^{3}$ calculated with the FP and MXW models. The two-dimensional map at base corresponds to the FP model.

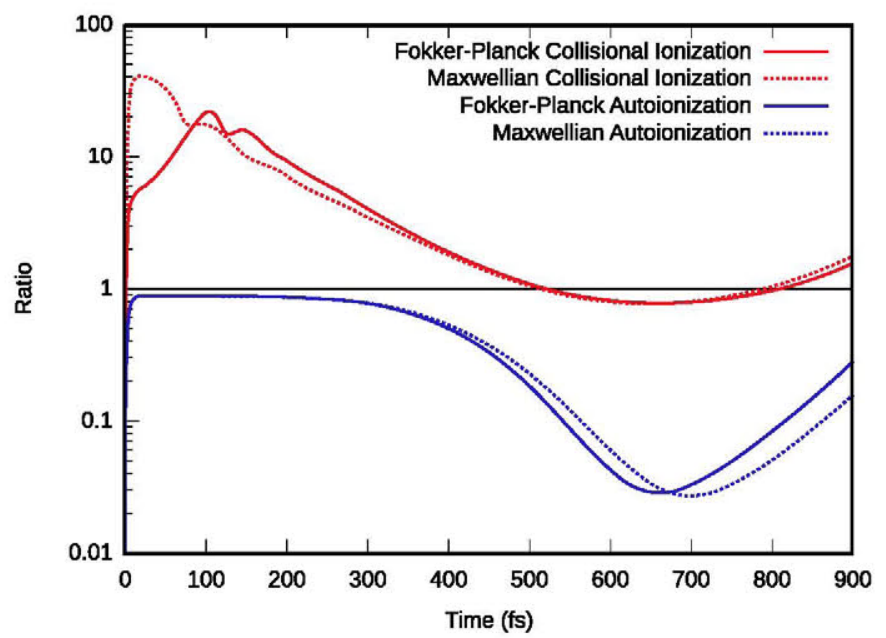

Fig. 5. Ratio of collisional ionization and autoionization total rate to the photoionization total rate at $10^{22}$ at $/ \mathrm{cm}^{3}$ for the FP and MXW models.

this state is reached in just $100 \mathrm{fs}$ in the FP model, as seen in Fig. 6. At higher density with the MXW model the ionization is even faster, $\eta^{\mathrm{Cl}}$ reaches its maximum at 20 fs yielding a collisional ionization rate that is a factor of 40 higher than the photoionization rate compared to a factor of 22 in the FP model, a fifth of the FP results, with a temperature minimum of $8.75 \mathrm{eV}$. The ionization state of $\bar{Z}=1$ is reached in $70 \mathrm{fs}$, with collisional ionization dominating the dynamics of the atomic populations from the beginning of the pulse. At 200 fs the electron density from the FP and MXW models cross and the FP shows a slightly larger ionization until the end of the pulse. As the electron distribution thermalizes in the FP model, $\eta^{\mathrm{CI}}$ in both models seem to match up.

The electronic response in the FP model is delayed when compared to the MXW model, and because of this the collisional ionization in the MXW model dominates when the intensity is still low. When collisions start to dominate in the FP model, the intensity is a few times higher than in the MXW case, which translates in a higher rate of high-energy photoelectron production which produces an increment in the electron temperature. Also, the mean ionization state that produce these absorptions in the FP case is lower, which can be observed in Fig. 9, producing photoelectrons

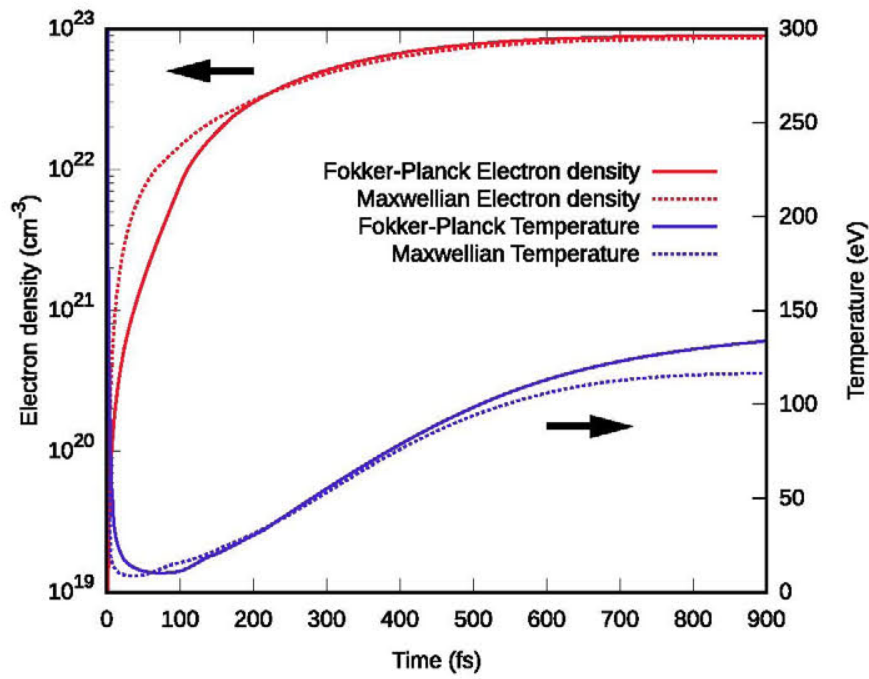

Fig. 6. Electron density and temperature evolution at $10^{22} \mathrm{at} / \mathrm{cm}^{3}$ for the FP and MXW models. 

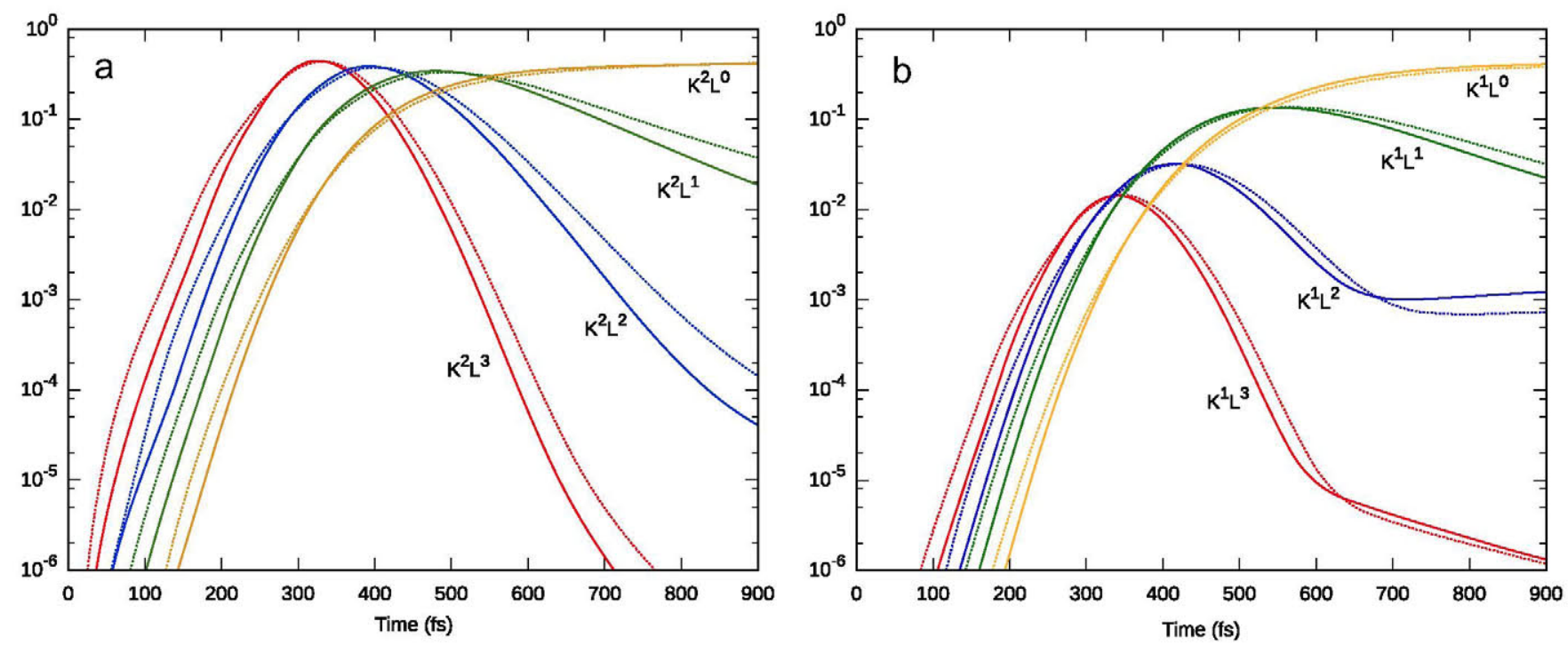

Fig. 7. Evolution of some full K-shell atomic levels (a) and levels with one hole in the K-shell (b) in the FP model (full line) and the MXW model (dotted line).

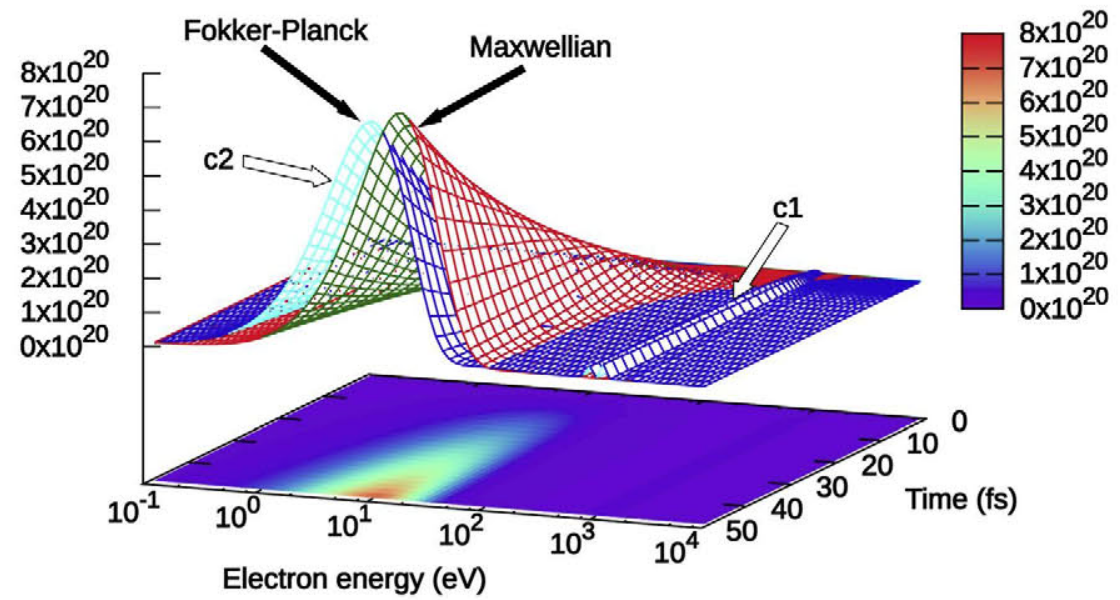

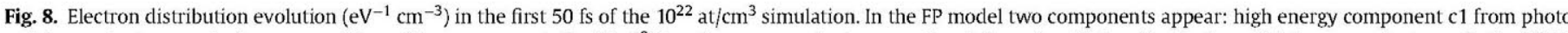

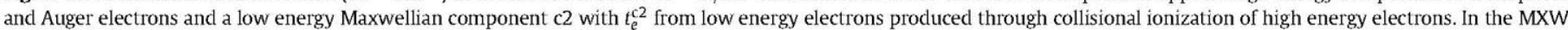
model the electron distribution is a mean of the low-energy and high-energy components.

with higher energies due to the fact that less ionized ions have lower ionization potentials (Tab. A.2 and A.3).

The MXW electron distribution's lower temperature produces higher collisional ionization rates, due to the higher overall overlap of the electron distribution and the collisional cross-sections, destroying autoionizing levels more efficiently than the FP model (600-900 fs). This induces a feedback, more autoionization increases the electron temperature which again decreases the overlap of the electron distribution with collisional cross-sections. This explains how the slightly higher $\eta^{\mathrm{CI}}$ in the MXW model (starting at $700 \mathrm{fs}$ ) prevents electron heating in the FP model. Almost at the same time, $\eta^{\mathrm{AI}}$ in the FP model exceeds the MXW value. The main contribution to the autoionization rate comes from the $\mathrm{K}^{1} \mathrm{~L}^{2}$ level. Fig. 7 shows the evolution of certain atomic levels with full K-shell and one hole in the K-shell.

To explain the differences in the first 200 fs Fig. 8 shows the electron distribution in both models in the first $50 \mathrm{fs}$. The FP model displays two main features, two high energy components from photoionized and Auger electrons, and a Maxwellian component due to low energy thermalized electrons from collisional ionization. Assuming thermalization (MXW) gives a shifted Maxwellian

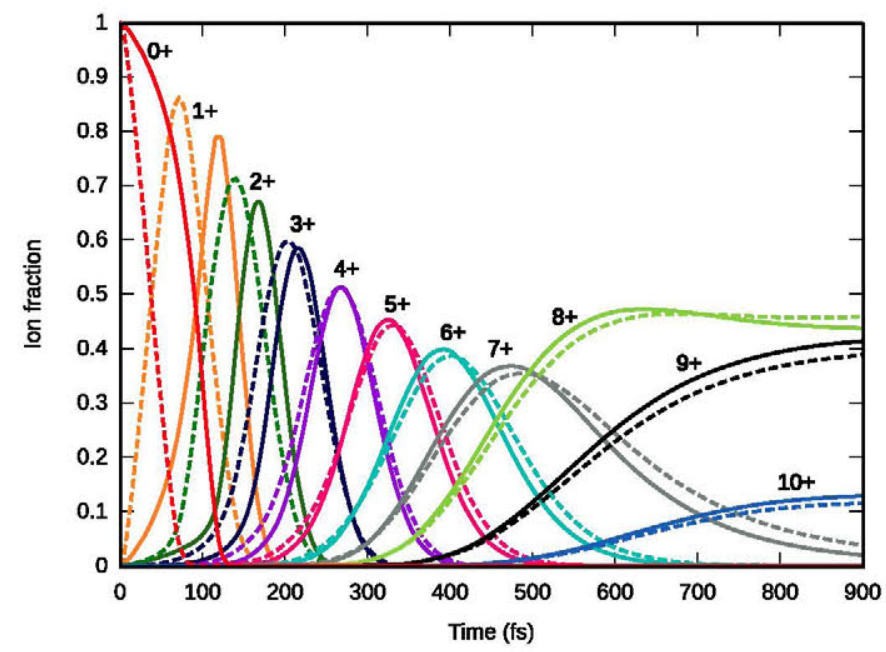

Fig. 9. Ion fractions evolution at $10^{22} \mathrm{at} / \mathrm{cm}^{3}$ from the FP model (full line) and the MXW model (dashed line). 
to higher energies, at approximately a mean of the thermalized and non-thermalized high energy electrons, enhancing the collisional ionization rates. Ion fractions $0+, 1+$ and $2+$ are depopulated faster in the MXW model because of enhanced overlap of the shifted Maxwellian with the collisional cross-sections.

This increases the temperature from 500 fs to the end of the pulse, due to the higher production of high energy electrons.

\section{Conclusions}

We have presented calculations of time-dependent atomic kinetics considering the electron distribution evolution during the irradiation of a sample with a high intensity X-ray laser. In the case where we use experimental density, the collisional rates are almost an order of magnitude lower than photo and autoionization. The non-Maxwellian nature of the electron distribution does not affect the atomic kinetics. At higher densities collisional processes play an important role, thermalization times are faster but not early in the pulse when the electron density is still low. The different collisional rates at the early times affect the ion population dynamics, leading to a increase in the electron temperature of $16 \%$ in the Fokker-Planck model (full treatment) compared to the Maxwellian model (instant thermalization).

\section{Acknowledgments}

We are grateful to the reviewers who helped clarify the presentation and improve the original text. This work has been supported by the Spanish Ministerio de Educación, Cultura y Deporte through program no. ENE2012-32108 and also by the European Commission project INREX-LASERLAB III.

\section{Appendix A. Autoionization and photoionization transitions}

Table A.1

Autoionization decay rates $A_{i j}\left(\mathrm{~s}^{-1}\right)$ and Auger electron energies $E_{e}(\mathrm{eV})$.

\begin{tabular}{llll}
\hline$i$ & $j$ & $A_{i j}$ & $E_{e}$ \\
\hline $\mathrm{K}^{0} \mathrm{~L}^{2}$ & $\mathrm{~K}^{1} \mathrm{~L}^{0}$ & $7.75 \times 10^{13}$ & 727 \\
$\mathrm{~K}^{1} \mathrm{~L}^{2}$ & $\mathrm{~K}^{2} \mathrm{~L}^{0}$ & $3.64 \times 10^{13}$ & 674 \\
$\mathrm{~K}^{0} \mathrm{~L}^{3}$ & $\mathrm{~K}^{1} \mathrm{~L}^{1}$ & $1.98 \times 10^{14}$ & 753 \\
$\mathrm{~K}^{1} \mathrm{~L}^{3}$ & $\mathrm{~K}^{2} \mathrm{~L}^{1}$ & $9.51 \times 10^{13}$ & 698 \\
$\mathrm{~K}^{0} \mathrm{~L}^{4}$ & $\mathrm{~K}^{1} \mathrm{~L}^{2}$ & $3.44 \times 10^{14}$ & 778 \\
$\mathrm{~K}^{1} \mathrm{~L}^{4}$ & $\mathrm{~K}^{2} \mathrm{~L}^{2}$ & $1.51 \times 10^{14}$ & 723 \\
$\mathrm{~K}^{0} \mathrm{~L}^{5}$ & $\mathrm{~K}^{1} \mathrm{~L}^{3}$ & $4.54 \times 10^{14}$ & 805 \\
$\mathrm{~K}^{1} \mathrm{~L}^{5}$ & $\mathrm{~K}^{2} \mathrm{~L}^{3}$ & $2.01 \times 10^{14}$ & 747 \\
$\mathrm{~K}^{0} \mathrm{~L}^{6}$ & $\mathrm{~K}^{1} \mathrm{~L}^{4}$ & $5.39 \times 10^{14}$ & 832 \\
$\mathrm{~K}^{1} \mathrm{~L}^{6}$ & $\mathrm{~K}^{2} \mathrm{~L}^{4}$ & $2.38 \times 10^{14}$ & 770 \\
$\mathrm{~K}^{0} \mathrm{~L}^{7}$ & $\mathrm{~K}^{1} \mathrm{~L}^{5}$ & $5.93 \times 10^{14}$ & 858 \\
$\mathrm{~K}^{1} \mathrm{~L}^{7}$ & $\mathrm{~K}^{2} \mathrm{~L}^{5}$ & $2.62 \times 10^{14}$ & 791 \\
$\mathrm{~K}^{0} \mathrm{~L}^{8}$ & $\mathrm{~K}^{1} \mathrm{~L}^{6}$ & $6.18 \times 10^{14}$ & 884 \\
$\mathrm{~K}^{1} \mathrm{~L}^{8}$ & $\mathrm{~K}^{2} \mathrm{~L}^{6}$ & $2.74 \times 10^{14}$ & 810 \\
\hline & & &
\end{tabular}

Table A.2

K-shell photoionization cross-section for a photon energy of $2 \mathrm{keV} \sigma_{i j}\left(\mathrm{~cm}^{2}\right)$ and photoelectron energies $E_{e}(\mathrm{eV})$.

\begin{tabular}{llll}
\hline$i$ & $j$ & $\sigma_{i j}$ & $E_{e}$ \\
\hline $\mathrm{K}^{1} \mathrm{~L}^{0}$ & $\mathrm{~K}^{0} \mathrm{~L}^{0}$ & $2.21 \times 10^{-20}$ & 637 \\
$\mathrm{~K}^{2} \mathrm{~L}^{0}$ & $\mathrm{~K}^{1} \mathrm{~L}^{0}$ & $3.89 \times 10^{-20}$ & 805 \\
$\mathrm{~K}^{1} \mathrm{~L}^{1}$ & $\mathrm{~K}^{0} \mathrm{~L}^{1}$ & $2.39 \times 10^{-20}$ & 698 \\
$\mathrm{~K}^{2} \mathrm{~L}^{1}$ & $\mathrm{~K}^{1} \mathrm{~L}^{1}$ & $3.83 \times 10^{-20}$ & 856 \\
$\mathrm{~K}^{1} \mathrm{~L}^{2}$ & $\mathrm{~K}^{0} \mathrm{~L}^{2}$ & $2.37 \times 10^{-20}$ & 752 \\
$\mathrm{~K}^{2} \mathrm{~L}^{2}$ & $\mathrm{~K}^{1} \mathrm{~L}^{2}$ & $3.79 \times 10^{-20}$ & 905 \\
$\mathrm{~K}^{1} \mathrm{~L}^{3}$ & $\mathrm{~K}^{0} \mathrm{~L}^{3}$ & $2.35 \times 10^{-20}$ & 802
\end{tabular}

Table A.2 (continued)

\begin{tabular}{lllr}
\hline$i$ & $j$ & $\sigma_{i j}$ & \multicolumn{1}{c}{$E_{e}$} \\
\hline $\mathrm{K}^{2} \mathrm{~L}^{3}$ & $\mathrm{~K}^{1} \mathrm{~L}^{3}$ & $3.88 \times 10^{-20}$ & 949 \\
$\mathrm{~K}^{1} \mathrm{~L}^{4}$ & $\mathrm{~K}^{0} \mathrm{~L}^{4}$ & $2.42 \times 10^{-20}$ & 849 \\
$\mathrm{~K}^{2} \mathrm{~L}^{4}$ & $\mathrm{~K}^{1} \mathrm{~L}^{4}$ & $3.94 \times 10^{-20}$ & 990 \\
$\mathrm{~K}^{1} \mathrm{~L}^{5}$ & $\mathrm{~K}^{0} \mathrm{~L}^{5}$ & $2.47 \times 10^{-20}$ & 891 \\
$\mathrm{~K}^{2} \mathrm{~L}^{5}$ & $\mathrm{~K}^{1} \mathrm{~L}^{5}$ & $3.99 \times 10^{-20}$ & 1027 \\
$\mathrm{~K}^{1} \mathrm{~L}^{6}$ & $\mathrm{~K}^{0} \mathrm{~L}^{6}$ & $2.51 \times 10^{-20}$ & 928 \\
$\mathrm{~K}^{2} \mathrm{~L}^{6}$ & $\mathrm{~K}^{1} \mathrm{~L}^{6}$ & $4.02 \times 10^{-20}$ & 1062 \\
$\mathrm{~K}^{1} \mathrm{~L}^{7}$ & $\mathrm{~K}^{0} \mathrm{~L}^{7}$ & $2.55 \times 10^{-20}$ & 960 \\
$\mathrm{~K}^{2} \mathrm{~L}^{7}$ & $\mathrm{~K}^{1} \mathrm{~L}^{7}$ & $4.05 \times 10^{-20}$ & 1094 \\
$\mathrm{~K}^{1} \mathrm{~L}^{8}$ & $\mathrm{~K}^{0} \mathrm{~L}^{8}$ & $2.58 \times 10^{-20}$ & 988 \\
$\mathrm{~K}^{2} \mathrm{~L}^{8}$ & $\mathrm{~K}^{1} \mathrm{~L}^{8}$ & $4.06 \times 10^{-20}$ & 1123 \\
\hline
\end{tabular}

Table A.3

L-shell photoionization cross-section for a photon energy of $2 \mathrm{keV} \sigma_{i j}\left(\mathrm{~cm}^{2}\right)$ and photoelectron energies $E_{e}(\mathrm{eV})$.

\begin{tabular}{llll}
\hline $\mathrm{i}$ & $\mathrm{j}$ & $\sigma_{i j}$ & $E_{e}$ \\
\hline $\mathrm{K}^{0} \mathrm{~L}^{1}$ & $\mathrm{~K}^{0} \mathrm{~L}^{0}$ & $8.20 \times 10^{-22}$ & 1659 \\
$\mathrm{~K}^{1} \mathrm{~L}^{1}$ & $\mathrm{~K}^{1} \mathrm{~L}^{0}$ & $6.36 \times 10^{-22}$ & 1720 \\
$\mathrm{~K}^{0} \mathrm{~L}^{2}$ & $\mathrm{~K}^{0} \mathrm{~L}^{1}$ & $1.31 \times 10^{-21}$ & 1705 \\
$\mathrm{~K}^{2} \mathrm{~L}^{1}$ & $\mathrm{~K}^{2} \mathrm{~L}^{0}$ & $5.68 \times 10^{-22}$ & 1771 \\
$\mathrm{~K}^{1} \mathrm{~L}^{2}$ & $\mathrm{~K}^{1} \mathrm{~L}^{1}$ & $1.17 \times 10^{-21}$ & 1758 \\
$\mathrm{~K}^{0} \mathrm{~L}^{3}$ & $\mathrm{~K}^{0} \mathrm{~L}^{2}$ & $1.80 \times 10^{-21}$ & 1746 \\
$\mathrm{~K}^{2} \mathrm{~L}^{2}$ & $\mathrm{~K}^{2} \mathrm{~L}^{1}$ & $1.04 \times 10^{-21}$ & 1807 \\
$\mathrm{~K}^{1} \mathrm{~L}^{3}$ & $\mathrm{~K}^{1} \mathrm{~L}^{2}$ & $1.60 \times 10^{-21}$ & 1796 \\
$\mathrm{~K}^{0} \mathrm{~L}^{4}$ & $\mathrm{~K}^{0} \mathrm{~L}^{3}$ & $2.20 \times 10^{-21}$ & 1784 \\
$\mathrm{~K}^{2} \mathrm{~L}^{3}$ & $\mathrm{~K}^{2} \mathrm{~L}^{2}$ & $1.39 \times 10^{-21}$ & 1840 \\
$\mathrm{~K}^{1} \mathrm{~L}^{4}$ & $\mathrm{~K}^{1} \mathrm{~L}^{3}$ & $1.90 \times 10^{-21}$ & 1831 \\
$\mathrm{~K}^{0} \mathrm{~L}^{5}$ & $\mathrm{~K}^{0} \mathrm{~L}^{4}$ & $2.45 \times 10^{-21}$ & 1823 \\
$\mathrm{~K}^{2} \mathrm{~L}^{4}$ & $\mathrm{~K}^{2} \mathrm{~L}^{3}$ & $1.65 \times 10^{-21}$ & 1872 \\
$\mathrm{~K}^{1} \mathrm{~L}^{5}$ & $\mathrm{~K}^{1} \mathrm{~L}^{4}$ & $2.12 \times 10^{-21}$ & 1864 \\
$\mathrm{~K}^{0} \mathrm{~L}^{6}$ & $\mathrm{~K}^{0} \mathrm{~L}^{5}$ & $2.62 \times 10^{-21}$ & 1858 \\
$\mathrm{~K}^{2} \mathrm{~L}^{5}$ & $\mathrm{~K}^{2} \mathrm{~L}^{4}$ & $1.84 \times 10^{-21}$ & 1902 \\
$\mathrm{~K}^{1} \mathrm{~L}^{6}$ & $\mathrm{~K}^{1} \mathrm{~L}^{5}$ & $2.28 \times 10^{-21}$ & 1895 \\
$\mathrm{~K}^{0} \mathrm{~L}^{7}$ & $\mathrm{~K}^{0} \mathrm{~L}^{6}$ & $2.74 \times 10^{-21}$ & 1891 \\
$\mathrm{~K}^{2} \mathrm{~L}^{6}$ & $\mathrm{~K}^{2} \mathrm{~L}^{5}$ & $1.98 \times 10^{-21}$ & 1930 \\
$\mathrm{~K}^{1} \mathrm{~L}^{7}$ & $\mathrm{~K}^{1} \mathrm{~L}^{6}$ & $2.38 \times 10^{-21}$ & 1923 \\
$\mathrm{~K}^{0} \mathrm{~L}^{8}$ & $\mathrm{~K}^{0} \mathrm{~L}^{7}$ & $2.80 \times 10^{-21}$ & 1921 \\
$\mathrm{~K}^{2} \mathrm{~L}^{7}$ & $\mathrm{~K}^{2} \mathrm{~L}^{6}$ & $2.07 \times 10^{-21}$ & 1955 \\
$\mathrm{~K}^{1} \mathrm{~L}^{8}$ & $\mathrm{~K}^{1} \mathrm{~L}^{7}$ & $2.44 \times 10^{-21}$ & 1949 \\
$\mathrm{~K}^{2} \mathrm{~L}^{8}$ & $\mathrm{~K}^{2} \mathrm{~L}^{7}$ & $2.11 \times 10^{-21}$ & 1977 \\
\hline & & & \\
& & & \\
& & &
\end{tabular}

\section{References}

1] H.-K. Chung, M.H. Chen, R.W. Lee, High Energy Density Phys. 3 (2007) 57

[2] S.B. Hansen, et al., High Energy Density Phys. 3 (2007) 109.

[3] R. Florido, et al., Phys. Rev. E 80 (2009) 056402.

[4] N.R. Pereira, K.G. Whitney, Phys. Rev. A 38 (1988) 319.

[5] T. Ditmire, Phys. Rev. E 54 (1996) 6735.

[6] S. Nayakshin, F. Melia, Astrophys. J. Suppl. Ser. 114 (1998) 269.

[7] J. Abdallah Jr., et al., Phys. Rev. A 68 (2003) 063201.

[8] L. Young, et al., Nature 466 (2010) 56.

[9] S.M. Vinko, et al., Nature 482 (2012) 59.

[10] F. de Gaufridy, et al., High Energy Density Phys. 7 (2011) 294.

[11] E. Oliva, et al., Nat. Photonics 6 (2012) 764.

[12] S.P. Hau-Riege, et al., Phys. Rev. E 69 (2004) 051906.

[13] A.G. de la Varga, et al., High Energy Density Phys. 7 (2011) 163.

[14] M.F. Gu, Astrophys. J. 582 (2003) 1241.

[15] A. Bar-Shalom, et al., Phys. Rev. A 40 (1989) 3183.

[16] R.M. More, J. Quant. Spectrosc. Radiat. Transfer 27 (1982).

[17] H.A. Scott, S.B. Hansen, High Energy Density Phys. 6 (2010) 39.

[18] D.H. Sampson, H.L. Zhang, Astrophys. J. 335 (1988) 516.

[19] H.A. Kramers, Philos. Mag 46 (1923) 836

[20] A. Burgess, M.C. Chidichimo, Mon. Not. R. Astron. Soc. 203 (1983) 1269.

[21] H.-K. Chung, et al., High Energy Density Phys. 1 (2005) 3.

[22] R.E.H. Clark, et al., Astrophys. J. 381 (1991) 597.

[23] H.-K. Chung, R.W. Lee, M.H. Chen, High Energy Density Phys. 3 (2007) 342

[24] S.J. Rose, High Energy Density Phys. 5 (2009) 23.

[25] S.D. Cohen, A.C. Hindmarsh, Comput. Phys. 10 (2) (1996) 138. 